\title{
Photoinduced Charge-Transfer Reactions of Tricyanoethylene with Aromatic Hydrocarbon Electron Donors. II. Semiempirical Studies of Naphthalene-Tricyanoethylene Exciplex
}

\author{
I. Deperasińska and J. Prochorow \\ Institute of Physics, Polish Academy of Sciences \\ al. Lotników 32/46, 02-668 Warsaw, Poland
}

(Received August 26, 2002)

\begin{abstract}
In this work we present the results of semiempirical AM1 calculations of the energy of electron-donor-acceptor system of naphthalene-tricyanoethylene complex in the ground and excited state with full optimization of the complex geometry. It was found that a ground state of the complex under consideration is stabilized almost exclusively by the electrostatic and Van der Waals interactions (presumably without charge-transfer resonance interaction). Its optimized equilibrium configuration has almost perpendicular head-to-face orientation of component molecules while in an excited state it is practically a charge-transfer electronic configuration with almost sandwich-like arrangement of both molecules. The energies of vertical electronic transitions between relevant ground and excited states and other spectroscopic parameters for these transitions were calculated with the use of ZINDO/S method and were compared with results of recent experimental observations of naphthalene-tricyanoethylene exciplex. This comparison led to a satisfactory explanation of the spectral properties and to a deeper insight into the kinetic parameters of the electron-donor-acceptor system under study.
\end{abstract}

PACS numbers: 82.30.Fi, 34.50.Gb

\section{Introduction}

Recently, in the course of emission studies of photoinduced charge-transfer reactions [1] for a series of aromatic hydrocarbon electron donors with tricyanoethylene (TRCNE), a strong electron acceptor from the family of cyanoethylenes [2], 
we have found that an electron-donor-acceptor (EDA) system of naphthalene (NP) and TRCNE seems to be somewhat peculiar among the other systems under study. Similarly to other electron donors of methylated benzenes family, NP forms relatively weak ground-state-stable charge-transfer or EDA complexes with TRCNE $[2,3]$, for which a characteristic CT absorption band can be observed; in nonpolar solvent direct excitation within this band leads to a very weak CT fluorescence [1]. Furthermore, we have found that TRCNE acts as a strong quencher of molecular fluorescence of NP, and that the mechanism of this bimolecular quenching reaction involves (or proceeds via) the formation of excited CT state, or exciplex. Fluorescence of NP-TRCNE exciplex displays a huge red-shift upon an increase of solvent polarity (of ca. $5000 \mathrm{~cm}^{-1}$ between $n$-hexane, $-\varepsilon=1.89$, and dichloromethane $-\varepsilon=9.6$, solutions) thus evidencing a large electric dipole moment of excited CT state. Briefly speaking, NP-TRCNE belongs to the wide class of EDA systems in which an interaction between electron-donor (D) and electron-acceptor (A) molecules results in a dynamic equilibrium, $\mathrm{D}+\mathrm{A} \rightleftharpoons(\mathrm{DA})$, of the formation of ground-state stable EDA complex (which by Mulliken's definition is stabilized due to the resonance interaction with its ionic $\left(\mathrm{D}^{+} \mathrm{A}^{-}\right)$charge-transfer state [4]), however, its excited CT state can be reached (or created) via two routes, either upon direct excitation of (DA) complex in its inherent CT absorption band:

$$
(\mathrm{DA})+h \nu_{\mathrm{CT}} \rightarrow\left(\mathrm{D}^{+} \mathrm{A}^{-}\right)
$$

or via an exciplex formation route, i.e. an electron transfer between excited species (either $\mathrm{D}^{*}$ or $\mathrm{A}^{*}$ ) and its EDA counterpart in the ground state, in a diffusion-controlled bimolecular fluorescence quenching reaction:

$$
\mathrm{D}+h \nu_{\mathrm{D}} \rightarrow \mathrm{D}^{*}+\mathrm{A} \rightarrow\left(\mathrm{D}^{+} \mathrm{A}^{-}\right)
$$

Detailed steady-state and time-resolved emission studies of monomer-exciplex equilibrium (2) performed for NP-TRCNE system in nonpolar solvent show that this EDA system approaches the kinetic and thermodynamic limits under which exciplex formation is controlled exclusively by the diffusion (without contribution of thermal dissociation of exciplex to the kinetics) and that the physical meaning of the decay parameters, that describe exciplex fluorescence, is reversed [1]. The presence of both equilibria (1) and (2) under conditions of liquid solution for a given EDA system, although recognized at quite early stages of exciplex' investigations [5], is still far from being fully understood, although its kinetical aspects of their competition (sometimes of important implications) were investigated and classified to some extent [6-8]. In the present case of NP-TRCNE system, the ground-state equilibrium has turned out to have no effects on excited state equilibria (which in part is due to a large separation between molecular (naphthalene) fluorescence band and exciplex (or CT) fluorescence band). 


\section{Comparative review and problem formulation}

When all the above-listed experimental facts for NP-TRCNE system are confronted with relevant data, available for its closest neighbors in the cyanoethylene family of electron-acceptors, i.e. with a rather rich bunch of data existing for tetracyanoethylene (TCNE) [9-14] and the only available (but otherwise reliable) data for fumaronitrile (trans-1,2-dicyanoethylene) $[15,16]$, then differences between this EDA system and those with participation of either TCNE or fumaronitrile (as an electron-acceptors) seem to be obvious and appealing.

TCNE is known to be the strongest organic electron-acceptor molecule and as such readily forms a variety of ground-state-stable EDA complexes of relatively high stabilities and high association constants (for a review see for instance [17]). However, none of its EDA complexes is fluorescent in liquid solution, even in nonpolar solvent, upon direct excitation within the CT absorption band (up to now, the only known example of such fluorescence is that of TCNE-hexamethylbenzene with a weak fluorescence in near-infrared region [14]). On the other hand CT fluorescence of TCNE EDA complexes with various organic aromatic donors in low-temperature rigid media [10-12] and under special conditions of jet-cooling in supersonic molecular beam [13] was found to be efficient. It seems to be well confirmed that the lack of CT fluorescence of EDA complexes of TCNE in nonpolar liquid solutions is due to the very efficient non-radiative processes operating in the course of relaxation of excited Franck-Condon state of different orientational isomers toward equilibrated excited CT state $[7,11,12]$, as well as, due to the inherent very high probability of radiationless transitions operating in equilibrated CT state in liquid solution [12]. In polar solvents, solvent-assisted and very fast formation of separated (solvated) ion pairs of the donor and acceptor as well as the charge recombination reaction of the geminate ion pairs, $D_{S}^{+} \ldots A_{S}^{-}$, are the main routes which overwhelm radiative decay of excited EDA complex [18]. In fluorescence quenching reactions TCNE acts as a very strong quencher of fluorescence of all aromatic electron donors, without exciplex fluorescence being detected even in nonpolar solvents. Nevertheless, the electron transfer reaction (and exciplex mechanism) between the donor and acceptor as an initial step of fluorescence quenching reaction seems to be well established by various femto-picosecond transient absorption experiments [19], although details of the competition between photoinduced charge separation, charge recombination in geminate ion pairs and formation of transient nonfluorescent exciplex state in solvents of different polarities still remain a subject to examination.

In the case of fumaronitrile (dicyanoethylene) - the third member in cyanoethylene family - the steady-state emission studies of its quenching of fluorescence of various polycondensed aromatic hydrocarbons in solvents of different polarities, have clearly demonstrated the formation of exciplex. The correlation between the energy of exciplex fluorescence maxima and the ionization potential of the aromatic hydrocarbon donors, as well as the red-shift of exciplex fluores- 
cence maxima upon an increase of solvent polarity have been observed [15]. Furthermore, in some cases, at relatively high concentrations of the components, a weak charge-transfer interaction in the ground state could have been also traced and in the case of acenaphthene-fumaronitrile an observation of a very weak charge-transfer fluorescence (as a result of excitation within the CT absorption band) have been reported. In the case of fumaronitrile-hexamethylbenzene EDA complex the position of CT fluorescence and of its intensity (yield) are both very strongly dependent on solvent polarity [16]. We have examined quantitatively fluorescence quenching in the case of naphtalene-fumaronitrile exciplex in $n$-hexane at different temperatures and compared the data with relevant data for NP-TRCNE case. Such a comparison (cf. Table I in Ref. [1]) shows that the quenching rate constant, $k_{\mathrm{q}}$, of molecular fluorescence of naphthalene by TRCNE, in $n$-hexane solution at room temperature, is larger by the factor of 2 from that for fumaronitrile. Furthermore, $k_{\mathrm{q}}$ for NP-TRCNE is increasing with increasing temperature, while $k_{\mathrm{q}}$ for naphthalene-fumaronitrile system is decreasing with an increase of temperature (at $328 \mathrm{~K}$ they differ by the factor of 4 ).

As a brief summary of this short review of vital (although selected) experimental data on the interaction of TCNE, TRCNE, and fumaronitrile electron-acceptor molecules with aromatic hydrocarbon donors in liquid solution, we notice that: (a) TCNE which is the strongest electron acceptor in this homologous series readily forms firmly strong ground-state-stable EDA complexes with a very distinct CT absorption band, however, for all practical purposes its CT excited state is nonfluorescent and the same is true when the excited CT state is formed in the course of photoinduced charge-transfer reaction (reaction scheme 2) - no exciplex emission can be observed; (b) TRCNE forms weak ground-state EDA complexes with aromatic hydrocarbons and weak CT emission can be excited and observed at least in nonpolar solvents (it fades away fast with an increase of solvent polarity); in photoinduced charge-transfer reactions it acts as a fairly strong quencher of molecular donor's fluorescence, but the exciplex emission is easily observed only in nonpolar solvents (in the case of NP-TRCNE system the monomer-exciplex equilibrium follows two-state photokinetic scheme and displays a peculiar kinetic case of the reversed physical meaning of the decay parameters of exciplex emission); (c) Fumaronitrile forms very weak EDA complexes and much easier detectable exciplexes, but its fluorescence quenching ability is clearly smaller than that of TRCNE.

We must stress, however, that although at first glance these conclusions seem to follow the order of decreasing electron-accepting ability of molecules under consideration, this is not completely true. This is revealed by a very simple test with the use of semiempirical Mulliken's relation [20] between energy of CT transition, $h \nu_{\mathrm{CT}}$, the ionization potential of the donor, $I_{\mathrm{D}}$, and the electron affinity of acceptor, $E_{\mathrm{A}}$ :

$$
h \nu_{\mathrm{CT}}=I_{\mathrm{D}}-E_{\mathrm{A}}+C,
$$


where the $C$ term includes energy of intermolecular interactions in the ground state (Van der Waals, dipole-dipole, etc.) as well as in the excited state (in this case overwhelmed by the energy of Coulombic attraction); in deriving Eq. (3) it is also assumed that both D and A molecules are neutral in the ground state. Relation (3) shows that in the case of naphthalene $\left(I_{\mathrm{D}}=8.18 \mathrm{eV}[21]\right)$, expected energy difference of CT transitions for its complexes with TCNE $\left(E_{\mathrm{A}}=2.88 \mathrm{eV}[22]\right)$ and with TRCNE $\left(E_{\mathrm{A}}=2.41 \mathrm{eV}[23]\right)$, should be of the order of $\sim 3800 \mathrm{~cm}^{-1}$ (with the assumption that the $C$ term in (3) is the same for both complexes). Combinations of available experimental data (not strictly comparable, as the data for NP-TCNE are those in liquid solution in $\mathrm{CCl}_{4}$ [24] and in PMMA matrix [25], while NP-TRCNE data are those for $n$-hexane solution [1]), lead to the value of $\Delta h \nu_{\mathrm{CT}}=h \nu_{\mathrm{CT}}(\mathrm{NP}-\mathrm{TRCNE})-h \nu_{\mathrm{CT}}(\mathrm{NP}-\mathrm{TCNE}) \approx 6000 \mathrm{~cm}^{-1}$.

Such a big difference between energies of CT transitions may indicate that the $C$ term in (3) must be distinctly different in both complexes. This might be due to the violation of the assumption that both molecules, D and A, are neutral in their ground states (which was taken for granted in the above-mentioned test), but other causes of this difference are also possible. In any case, however, one could expect that such big difference between CT transition energies for this two complexes would be reflected by differences in their ground-state (and/or excited-state) structures. In accordance with the requirements of Mulliken's "overlap and orientation principle" [20], most of the TCNE complexes with aromatic hydrocarbon donors are known to have sandwich-like structure with molecular planes of the donor and acceptor in parallel, although not necessarily in central arrangement, which is also true for NP-TCNE complex [25, 26]. However, this not necessarily must be true for complexes (and or exciplexes) of TRCNE molecule which has no symmetry elements (besides an identity) and presumably must possess non-zero electric dipole moment in the ground state.

The present work is aimed at theoretical studies of geometrical structure of TRCNE molecule and of NP-TRCNE complex in the ground and excited state. The electronic structure of the complex is also to be found. We shall calculate and examine the energies of electronic transitions (relevant to absorption and emission of the complex) for relevant optimized ground and excited structures, as well as the other spectral characteristics, which are to be compared with experimental observations. These results are also expected to give some insight into the kinetic parameters of excited complex related either to relaxation or decay of NP-TRCNE exciplex.

\section{Computational methods}

The geometries of NP-TRCNE complex in the ground and excited states (and separately of both isolated, non-interacting molecules) were optimized within the framework of semiempirical AM1 method [27], with configuration interaction for microstates, C.I. $=2$. All internal coordinates for the donor and acceptor 
molecules and all six intermolecular coordinates of their EDA complex were taken into account in the procedure of geometry optimizations.

The use of configuration interaction in C.I. $=2$ option is equivalent to Mulliken's theory of charge-transfer state in its classical form of the "whole-complex-molecular-orbital description" [20], in which configuration interaction includes two orbitals, i.e. HOMO and LUMO localized on the donor and on the acceptor molecule, respectively. This approximation has been very successfully used in the description of the properties of many EDA systems [20,28], and in general it can be used for those systems, for which HOMO and LUMO orbitals are well separated from the rest of molecular orbitals of the complex. This is the case of NP-TRCNE system in which the both, HOMO and LUMO, are separated from other molecular orbitals by more than $0.5 \mathrm{eV}$ (as found either by AM1 or by ZINDO/S methods).

Calculations of spectral properties, i.e. of the vertical transition energies (from ground-to-excited state and vice versa), the oscillator strengths, and the dipole moments, for AM1 optimized structures of NP-TRCNE system were performed with the use of ZINDO/S method including 201 singly excited configurations. The choice of ZINDO/S method was due to the fact that it is much better reproducing the HOMO-LUMO energy gap of NP-TRCNE complex than AM1 method; within the framework of ZINDO/S method this gap is $5.7 \mathrm{eV}$, while the corresponding value for $\mathrm{AM} 1$ is $6.9 \mathrm{eV}$ (and both are to be compared to the difference between ionization potential of the donor [21] and electron affinity of the acceptor [23]: $\left.I_{\mathrm{D}}-E_{\mathrm{A}}=5.77 \mathrm{eV}\right)$.

\section{Results of calculations and discussion}

\subsection{Tricyanoethylene molecule}

Tricyanoethylene is chemically similar to tetracyanoethylene (TCNE), and like TCNE it is $\pi$-acid, whose acid strength as compared to TCNE is decreased in correspondence to the change of relative electron-withdrawing character of cyano and hydrogen [2], as reflected by electron affinities of both molecules [22, 23] (and in basic accordance with spectral properties of their EDA complexes with aromatic hydrocarbons [1-4, 9, 10]; cf. Eq. (3) in Sec. 2).

Figure 1 shows the structure of TRCNE molecule, which is a planar structure - the plane of molecule contains all of its 8 chemical bonds: the central $\mathrm{C}=\mathrm{C}$ double bond, three $\mathrm{C}-\mathrm{C}$ single bonds, three $\mathrm{C} \equiv \mathrm{N}$ triple bonds and one $\mathrm{C}-\mathrm{H}$ single bond. The lengths of these bonds, as they come from AM1 optimized ground state structure of molecule are also given in Fig. 1. A substitution of the fourth cyano group in TRCNE molecule, which transforms it into TCNE molecule, leads to some minor "corrections" of the bond-lengths (e.g. $\mathrm{C}=\mathrm{C}$ bond acquires the length of $1.36 \AA$ as compared to $1.35 \AA$ in TRCNE, and the angle between $\mathrm{C}=\mathrm{C}$ bond and all four $\mathrm{C}-\mathrm{C}$ single bonds become equal to $122^{\circ}$, while in TRCNE these angles vary from $124^{\circ}$ to $121^{\circ}$ in the case of $\mathrm{C}-\mathrm{H}$ single bond). 


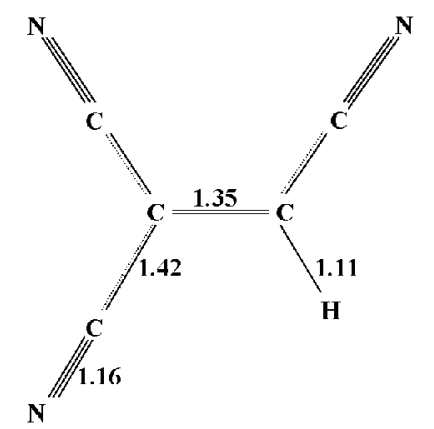

Fig. 1. Geometrical structure of tricyanoethylene (TRCNE) molecule. Numbers are the lengths of respective chemical bonds (in $\AA$ ).

However, there is a difference between TCNE and TRCNE molecules, which might be of importance from the point of view of charge-transfer reactions of both types, either photoinduced or due to the EDA complex formation in the ground state. TCNE molecule which contains four cyano groups is highly-symmetric ( $D_{2 h}$ point symmetry group) and is neutral in its ground state with electric dipole moment virtually equal to zero. On the other hand, TRCNE molecule which has no symmetry elements, is characterized by asymmetric distribution of the electronic charge over the molecule, which results in the net electric dipole moment. The results of the calculations of electrostatic potential field of TRCNE and its dipole moment are given in Fig. 2 . It is seen that the dipole moment vector $\mu_{\mathrm{TRCNE}}$ (of the magnitude of $2.37 \mathrm{D}$ ) lies in the molecular plane of TRCNE molecule and is directed across the central $\mathrm{C}=\mathrm{C}$ bond, at an angle of $49^{\circ}$, towards $\mathrm{H}$ atom.

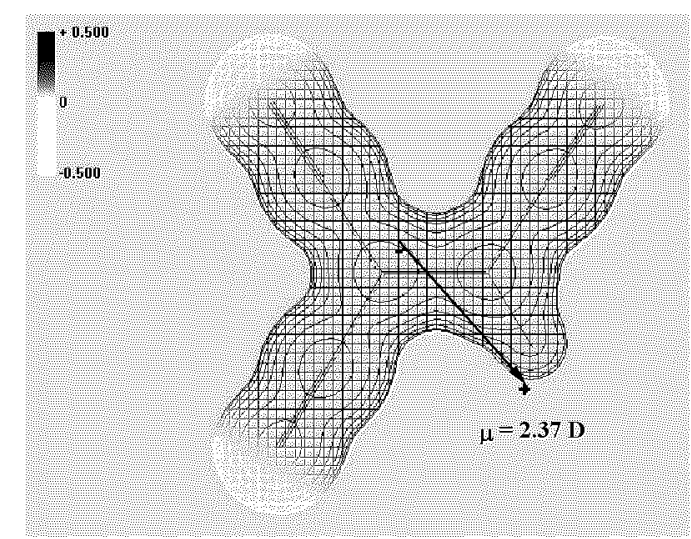

Fig. 2. Electrostatic potential field, produced by electronic charge distribution and nuclear charges (in e/ $\AA$ ) in TRCNE molecule in the ground state. Arrow represents the vector of electric dipole moment of the molecule (related to asymmetric electrostatic field). 


\subsection{Optimized equilibrium structures of NP-TRCNE electron-donor-acceptor system}

The equilibrium configurations of NP-TRCNE complex in the ground and excited state were computed as quasi-adiabatic states which result from the configuration interaction between the ground (DA) and $\left(\mathrm{D}^{+} \mathrm{A}^{-}\right)$electronic configurations for AM1 optimized isolated donor and acceptor molecule. The optimized equilibrium structures in the ground $\left(\boldsymbol{G}_{\mathrm{eq}}\right)$ and excited $\left(\boldsymbol{E}_{\mathrm{eq}}\right)$ state of NP-TRCNE complex are sketched in Fig. 3, and some selected parameters of their geometries are given in Table I.

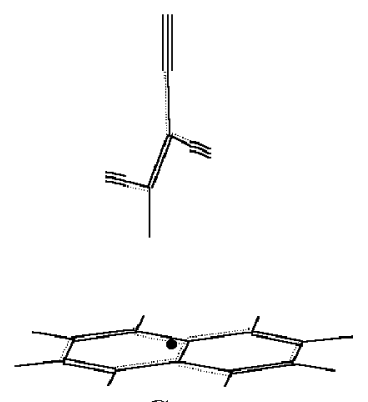

Geq

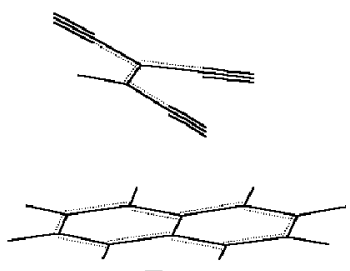

Eeq

Fig. 3. The AM1 optimized geometries of equilibrium structures in the ground $\left(\boldsymbol{G}_{\text {eq }}\right)$ and excited $\left(\boldsymbol{E}_{\mathrm{eq}}\right)$ states of NP-TRCNE electron-donor-acceptor system. (Black dot indicates the region where the extension of direction of the single $\mathrm{C}-\mathrm{H}$ bond of TRCNE would intersect the molecular plane of NP molecule).

TABLE I

Selected structural parameters for AM1 optimized geometry of equilibrium configurations of NP-TRCNE complex in the ground $\boldsymbol{G}_{\text {eq }}$ and excited $\boldsymbol{E}_{\mathrm{eq}}$ state, and of its component molecules.

\begin{tabular}{l|c|c}
\hline \hline \multicolumn{1}{c|}{ Structural parameters } & $\boldsymbol{G}_{\mathrm{eq}}$ & $\boldsymbol{E}_{\mathrm{eq}}$ \\
\hline Angle between NP and TRCNE molecular planes & $88^{\circ}$ & $16^{\circ}$ \\
\hline $\begin{array}{l}\text { Angle between } \mathrm{C}=\mathrm{C} \text { bond direction of NP and } \\
\text { molecular plane of TRCNE molecule }\end{array}$ & $35^{\circ}$ & $0^{\circ}$ \\
\hline $\begin{array}{l}\text { Perpendicular distance between H atom of TRCNE } \\
\text { and molecular plane of NP molecule }\end{array}$ & $2.58 \AA$ & $3.76 \AA$ \\
\hline Length of C = C bond in TRCNE molecule & $1.351 \AA$ & $1.394 \AA$ \\
\hline \begin{tabular}{l} 
Length of central C $=\mathrm{C}$ bond in NP molecule \\
\hline
\end{tabular} & $1.418 \AA$ & $1.434 \AA$
\end{tabular}

As we speculated in Sec. 2 - in view of what is known about the ground-state structures of EDA complexes of napthalene with typical organic acceptors (and this concerns as well the structures of most of EDA complexes of typical organic 
electron-acceptors with other aromatic hydrocarbon donors) - the geometry of $\boldsymbol{G}_{\text {eq }}$ structure has turned out to be evidently different from generally expected sandwich-like arrangement of donor, D, and acceptor, A, molecules in the complex unit (DA). Instead, it is a "head-to-face" almost perpendicular arrangement of molecular planes of both molecules (with an angle of $88^{\circ}$ between their molecular planes, cf. Tab. I). C - H single bond of TRCNE molecule is aimed at molecular plane of NP (or more precisely at one of NP's aromatic rings in a close vicinity of one of the $\mathrm{C}$ atoms of the central bond of aromatic skeleton of NP, in the place indicated by the black dot). The closest distance between both molecules is that of $2.58 \AA$ between $\mathrm{H}$ atom and molecular plane of TRCNE (cf. Table I). Hence, TRCNE molecule is inclined with an angle between its central $\mathrm{C}=\mathrm{C}$ bond and molecular plane of NP equal to $35^{\circ}$ (cf. Tab. I). We should notice also that the bond-lengths of TRCNE molecule in the optimized ground state $\boldsymbol{G}_{\text {eq }}$ structure of NP-TRCNE complex are not changed as compared with the bond-lengths of optimized structure of a free, isolated molecule (cf. Fig. 1 and Tab. I), and the same is true for NP molecules.

The binding (or stabilization) energy of $\boldsymbol{G}_{\text {eq }}$ equilibrium structure, calculated as a gain of energy relative to the sum of energies of isolated donor and acceptor molecule in their ground state, is $E_{\mathrm{G}}=480 \mathrm{~cm}^{1}(\approx 1.4 \mathrm{kcal} / \mathrm{mole})$. This is a small value, roughly by one order of magnitude smaller than the binding energies (or strictly speaking, known heats of formation) of the ground state of TCNE complexes with aromatic hydrocarbon donors in the gas phase [29, 30]). Hence, one could arrive at the conclusion that a NP-TRCNE CT complex in its ground state may not be stabilized by the "charge-transfer" contribution to its binding energy. Especially as such CT contribution, due to resonance between (DA) and $\left(\mathrm{D}^{+} \mathrm{A}^{-}\right)$ electronic structures, has a maximum value for a sandwich arrangement of EDA systems of acceptors and donors under consideration. Since TRCNE molecule, as found in the course of this studies, is a polar molecule with a relatively large dipole moment (of $2.37 \mathrm{D}$ ) and since in $\boldsymbol{G}_{\text {eq }}$ equilibrium structure of NP-TRCNE complex the vector of TRCNE dipole moment points towards the aromatic $\pi$-electrons system of NP molecule, the stabilization of NP-TRCNE complex in the ground state can be assured predominantly by the electrostatic or Van der Waals type of interactions, with a minor (or without) resonance CT contribution (although it should be born in mind that the distinction, between the contribution of "resonance CT interaction" and of "Van der Waals interactions" to the binding of very weak complex, may be a kind of semantic problem, as pointed on many occasions; see for instance [31]). And on top of that, one may not exclude the possibility of a weak hydrogen-bond formation between $\mathrm{H}$ atom of TRCNE and the $\pi$-electrons of aromatic skeleton system of NP molecule.

Equally important, although not fully unexpected result of the optimization procedure is a huge change (or reorganization) of geometry of optimized excited state $\boldsymbol{E}_{\text {eq }}$ equilibrium structure of NP-TRCNE system (as compared to the geom- 
etry of ground state $\boldsymbol{G}_{\text {eq }}$ equilibrium structure). As seen in Fig. 3 and revealed by the data of Tab. I, in excited state complex acquires an almost sandwich central structure - molecular planes of both molecules deviate from parallel orientation by $16^{\circ}$ only and the central $\mathrm{C}=\mathrm{C}$ bond of TRCNE is strictly parallel to the NP molecular plane (or to its short molecular axis), though it is slightly shifted (in its planar projection onto the molecular plane of NP) to the position closer to the center of the one of the two rings of aromatic skeleton of NP. Perpendicular separation of $\mathrm{C}=\mathrm{C}$ bond of TRCNE from the molecular plane of NP is $3.50 \AA$, but this time the closest distance between both molecules is that between $\mathrm{N}$ atoms of two $\mathrm{C} \equiv \mathrm{N}$ terminal cyano groups which equals $2.92 \AA$; the single $\mathrm{C}-\mathrm{H}$ bond points upward and is far from the molecular plane of naphthalene.

Another important point is that the optimization procedure leaves in this state both, D and A, molecules in geometries corresponding to optimized geometries of their radical ions. This is seen when bond-lengths of the donor and acceptor in the optimized $\boldsymbol{E}_{\text {eq }}$ structure are compared to relevant bond-lengths of their ions. Thus, the bond-length of the $\mathrm{C}=\mathrm{C}$ double bond of TRCNE of $1.394 \AA$ is larger than in the ground $\boldsymbol{G}_{\text {eq }}$ structure (cf. Tab. I), but it is fairly close to $1.401 \AA$ obtained for optimized TRCNE ${ }^{-}$anion. Similarly, the length of the central C $=\mathrm{C}$ bond of NP aromatic skeleton $-1.434 \AA$ is longer in the excited $\boldsymbol{E}_{\text {eq }}$ structure (cf. Tab. I), but is very close to $1.436 \AA$ obtained for the optimized structure of $\mathrm{NP}^{+}$cation. Hence, the excited state of NP-TRCNE EDA system has clearly an ionic electronic structure of a $\left(\mathrm{D}^{+} \mathrm{A}^{-}\right)$type, or in other words it is CT excited state. This is also indicated by the computed value of its dipole moment which has a magnitude of $15.94 \mathrm{D}$.

\subsection{Comparison of the calculated electronic transitions and experimental absorption and fluorescence spectra of NP-TRCNE system}

As concluded just above, the first excited singlet state of EDA system of NP-TRCNE is the $\left(\mathrm{D}^{+} \mathrm{A}^{-}\right) \mathrm{CT}$ excited state. However, this state cannot be reached directly by a vertical electronic transition (excitation) from the $\boldsymbol{G}_{\text {eq }}$ ground state of the complex. The vertical electronic transition from the ground state leads to the excited Franck-Condon state (of the same geometry as that of the ground state $\boldsymbol{G}_{\text {eq }}$ equilibrium structure), whose electric dipole moment, as derived from these computations, has a very large value of $23.73 \mathrm{D}$. In terms of multidimensional potential energy surface (PES) of excited state of NP-TRCNE complex, the excited Franck-Condon state has an excess of energy of $5570 \mathrm{~cm}^{-1}$ relative to the PES minimum, which corresponds to the equilibrium excited state $\boldsymbol{E}_{\text {eq }}$ structure. An inspection of the relaxation pathway along the excited state PES, from the excited Franck-Condon state toward the $\boldsymbol{E}_{\text {eq }}$ minimum, reveals no energy barriers for this relaxation.

A vertical electronic transition in opposite direction, from the equilibrated excited $\left(\mathrm{D}^{+} \mathrm{A}^{-}\right) \mathrm{CT}$ state (reached as a terminal equilibrated state of the relax- 
ation of Franck-Condon state) terminates in the ground state Franck-Condon state, whose energy is by $3675 \mathrm{~cm}^{-1}$ higher than the energy minimum of the ground state PES (the energy of the ground state $\boldsymbol{G}_{\text {eq }}$ equilibrium structure), or ca. $3200 \mathrm{~cm}^{-1}$ above the dissociation limit of the complex in the ground state. The computed dipole moment of the ground Franck-Condon state is 2.06 D.

The calculated energies of both above-described vertical electronic transitions: ground-to-excited and excited-to-ground state, can be compared with absorption (or fluorescence excitation) and fluorescence spectra of NP-TRCNE system, respectively. Such comparison is presented in Fig. 4.
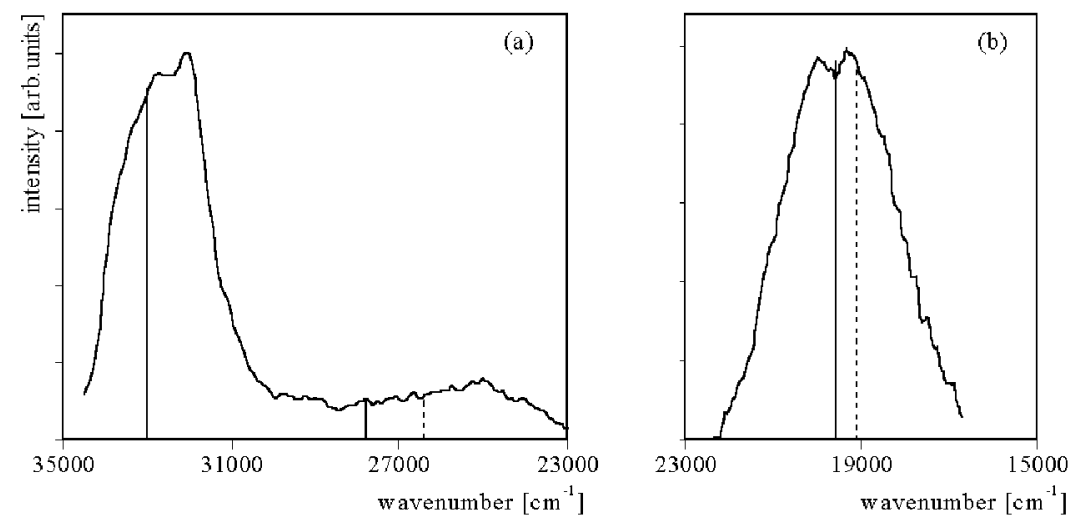

Fig. 4. (a) Exciplex fluorescence excitation spectrum of NP-TRCNE EDA system in $n$-hexane solution (reproduced after [1]). Solid vertical lines are the calculated energies of the two lowest vertical electronic transitions from the ground $S_{0}$ state, dotted line is the transition energy "corrected" for the influence of solvent (see the text for details). (b) Exciplex (and CT) fluorescence spectrum of NP-TRCNE EDA system in $n$-hexane solution (reproduced after [1]). Solid vertical line is the calculated energy of the vertical electronic transition from the excited $S_{1}$ state, dotted line is the transition energy "corrected" for the influence of solvent (see the text for details).

A fluorescence excitation spectrum of NP-TRCNE observed in $n$-hexane solution at room temperature is given in Fig. 4 a (for details of excitation and of monitoring of fluorescence and fluorescence excitation spectra see [1]). This spectrum consists of an intense molecular NP absorption band with a maximum at $c a .32200 \mathrm{~cm}^{-1}$ and a very flat and much weaker CT absorption band with a maximum located at $25000 \pm 500 \mathrm{~cm}^{-1}$.

The energies of vertical electronic transitions, calculated within the framework of ZINDO/S method, are represented by vertical lines and are collected in Table II, together with corresponding transition oscillator strengths. It is seen that the only one of the two calculated electronic transitions, from the ground $S_{0}$ singlet state to the two lowest excited singlet states $\left(S_{1}\right.$ and $\left.S_{2}\right)$, has the energy 
which is in fairly good ( $c a .2 \%$ difference only) agreement with the experimental spectrum. This $S_{0} \rightarrow S_{2}$ transition leads to an excited $S_{2}$ singlet state, which is of a locally excited (LE) character, or in other words which is an excited singlet state of the donor molecule, and as such, may be only very slightly or not at all modified by the formation of EDA complex (as it happens in the case of TCNE complexes [32]).

TABLE II

Results of ZINDO/S calculations of the energy $(\Delta E)$ of the lowest electronic transitions from the ground $S_{0}\left(\equiv \boldsymbol{G}_{\mathrm{eq}}\right)$ and from the excited $S_{1}(\equiv$ $\boldsymbol{E}_{\text {eq }}$ ) electronic states of NP-TRCNE and their oscillator strengths (f). For comparison the energy of maxima of observed [1] exciplex fluorescence excitation bands and of maximum of exciplex fluorescence band are given in parenthesis.

\begin{tabular}{c|c|c}
\hline \hline Electronic transition & $\Delta E\left(\mathrm{~cm}^{-1}\right)$ & $\mathrm{f}$ \\
\hline$S_{0} \rightarrow S_{1}$ & 27790 & 0.003 \\
& $(25000)$ & \\
$S_{0} \rightarrow S_{2}$ & 33010 & 0.029 \\
& $(32200)$ & \\
\hline$S_{1} \rightarrow S_{0}$ & 19560 & 0.0484 \\
& $(19600)$ &
\end{tabular}

There is, however, a relatively large discrepancy between the experimentally observed CT fluorescence excitation band in the region below $29000 \mathrm{~cm}^{-1}$ (which is fully equivalent to the CT absorption band) and the calculated energy of $S_{0} \rightarrow S_{1}$ transition (which is a CT transition, characterized by the oscillator strength for the transition that is by one order of magnitude smaller than the oscillator strength for the transition to LE singlet state $S_{2}$ (cf. Tab. II)). The calculated energy of the transition is shifted toward higher energies by $c a .2500-3000 \mathrm{~cm}^{-1}$, relative to the maximum of the CT absorption band located at $25000 \pm 500 \mathrm{~cm}^{-1}$ (more than $10 \%$ difference between experiment and calculations).

At this point we should take into account the fact that the calculated energies of the transitions are the "gas phase" energies of isolated molecules (or complex), while the experimental spectra refer to the solution. Especially in the case where the changes of the dipole moment between the ground and excited state are large, which is the case under consideration, the solvent-solute interactions can appreciably contribute to the energies of both states and by the same token to the energies of electronic transitions. For the determination of solvent effects and calculations of relevant solvent shifts of the absorption and fluorescence transitions we use the Mataga-Weller model $[33,34]$. The energy of a molecule (complex) in the solvent 
is the sum of its energy in the gas phase (i.e. energy of free, isolated molecule which actually is that obtained with AM1 method), and the energy of its solvation. The solvation energy can be approximated as a result of interaction between the permanent dipole of the complex and surrounding dielectric medium. The solvent shifts of the absorption and of the fluorescence transitions are given as:

$$
\begin{aligned}
& \Delta E_{\mathrm{a}}=E_{\mathrm{a}}^{\mathrm{sol}}-E_{\mathrm{a}}^{\mathrm{gas}}=\mu_{\mathrm{g}}\left(\mu_{\mathrm{g}}-\mu_{\mathrm{e}}\right) f_{\varepsilon}-\mu_{\mathrm{e}}\left(\mu_{\mathrm{e}}-\mu_{\mathrm{g}}\right) f_{n}, \\
& \Delta E_{\mathrm{f}}=E_{\mathrm{f}}^{\mathrm{sol}}-E_{\mathrm{f}}^{\mathrm{gas}}=\mu_{\mathrm{e}}\left(\mu_{\mathrm{g}}-\mu_{\mathrm{e}}\right) f_{\varepsilon}+\mu_{\mathrm{e}}\left(\mu_{\mathrm{e}}-\mu_{\mathrm{g}}\right) f_{n},
\end{aligned}
$$

with

$$
f_{\varepsilon}=\frac{2}{h c a^{3}} \frac{\varepsilon-1}{2 \varepsilon+1} \quad \text { and } \quad f_{n}=\frac{2}{h c a^{3}} \frac{n^{2}-1}{2 n^{2}+1},
$$

where $\varepsilon$ and $n$ are the solvent's dielectric constant and index of refraction, respectively; $\mu_{\mathrm{g}}$ and $\mu_{\mathrm{e}}$ are the respective dipole moments of the complex ground and excited state, which are actually combining in the transition under consideration (i.e. the ground, $\boldsymbol{G}_{\text {eq }}$, state and its Franck-Condon excited state in the case of $S_{0} \rightarrow S_{1}$ transition and the excited, $\boldsymbol{E}_{\mathrm{eq}}$, state and its Franck-Condon ground state, respectively). The parameter $a$ in $f_{\varepsilon}$ and $f_{n}$ functions is the radius of Onsager sphere which contains a complex unit, and these were estimated in the present calculations as $5.07 \AA$ and $4.72 \AA$ for $\boldsymbol{G}_{\text {eq }}$ and $\boldsymbol{E}_{\text {eq }}$ structures, respectively.

As seen in Fig. 4a, such a treatment of solvent effects shifts the energy of $S_{0} \rightarrow S_{1}$ vertical electronic transition toward lower energies by $\mathrm{ca} .1400 \mathrm{~cm}^{-1}$, which improves agreement between the results of calculations and experiment. Still, however, this correction to the transition energy is not large enough to account satisfactorily for the observed difference between the computed and experimental values. To this end, two additional factors might probably be taken into account. First of all, it is possible that an optimized equilibrium structure (in this case the ground state $\boldsymbol{G}_{\text {eq }}$ equilibrium structure), which is a quite loosely-bound entity (cf. discussion in Sec. 4.2), may be much more affected by the interaction with solvent molecules (which may include both, the dielectric shift and the solvent cage effect [35]), and its geometry would not be retained in a solution. The second possibility is that, due to the weak binding of the ground state complex, instead of one equilibrium configuration there is a variety of different ground state conformers (or orientational isomers), of slightly different energies, and those are contributing to the observed wide and flat CT fluorescence excitation band. However, up to now, NP-TRCNE EDA system is the first studied in details and verification of above-mentioned possibilities (and probably some others not yet realized) must await more results of experiments to be carried out along that line.

Contrary to the $S_{0} \rightarrow S_{1}$ transition, the calculated energy of $S_{1} \rightarrow S_{0}$ vertical electronic transition which is to be compared with the CT fluorescence spectrum (or exciplex fluorescence) fits closely the experimental findings. This is illustrated 
in Fig. 4b, where the computed energy of this transition is compared with the observed exciplex fluorescence band in $n$-hexane solution (which, as previously shown, is fully equivalent to CT fluorescence spectrum excited within the absorption CT band - cf. Fig. 7 in Ref. [1]). The maximum of the fluorescence band is $19600 \pm 400 \mathrm{~cm}^{-1}$, whereas the calculated energy of the transition is $19560 \mathrm{~cm}^{-1}$ (cf. Tab. II). Upon correction for solvent effects this energy shifts toward lower energies by $400 \mathrm{~cm}^{-1}$ and thus practically fits the error limit for the position of the fluorescence band maximum. In view of this excellent agreement between the experimental and calculated results, we may conclude that the equilibrated (relative to the solvent), excited $\left(\mathrm{D}^{+} \mathrm{A}^{-}\right) \mathrm{CT}$ state of NP-TRCNE system must have the electronic and geometrical structure, which in fact closely corresponds to the AM1 optimized excited state equilibrium configuration $\boldsymbol{E}_{\text {eq }}$.

The last conclusion can be considered as a strong support of the reliability of other spectroscopic parameters that were obtained in the present computations (within the framework of ZINDO/S method). Probably the most important finding of these calculations is that concerning oscillator strength of radiative $S_{1} \rightarrow S_{0}$ transition. Its relatively large value (cf. Tab. II) is due to the deviation of the sandwich-like $\boldsymbol{E}_{\text {eq }}$ equilibrium structure from strictly parallel orientation of molecular planes of the donor and acceptor molecules (which by virtue of Mulliken's "overlap and orientation principle" [20] would lead to the oscillator strength equal to zero)

We can try to use the obtained value of the oscillator strength of radiative $S_{1} \rightarrow S_{0}$ transition for estimations of kinetic parameters of $S_{1}$ excited CT state. With a semiempirical formula which relates the oscillator strength $f$ of a given electronic transition to the natural radiative lifetime $\tau_{0}$ of the initial state [36]:

$$
\tau_{0} \approx \frac{1.5}{\nu_{\max }^{2} f}
$$

where $\nu_{\max }$ is the wavenumber of the maximum of the fluorescence band. With $\mathrm{f}=0.0485$ ( $\mathrm{cf}$. Tab. II) we find that the rate constant $k_{\mathrm{r}}\left(=1 / \tau_{0}\right)$ of radiative transition from $S_{1}$ is $k_{\mathrm{r}}=1.24 \times 10^{7} \mathrm{~s}^{-1}$. The experimentally determined rate constant for the overall decay (the sum of the rate constants for radiative, $k_{\mathrm{r}}$, and nonradiative, $k_{\mathrm{nr}}$, decay) of NP-TRCNE exciplex is $k_{\mathrm{p}}=k_{\mathrm{r}}+k_{\mathrm{nr}}=2.76 \times 10^{8} \mathrm{~s}^{-1}$ (cf. Tab. III in Ref. [1]). Now combining these two values we find that the quantum yield $\phi$ of exciplex fluorescence of NP-TRCNE is: $\phi=k_{\mathrm{r}} /\left(k_{\mathrm{r}}+k_{\mathrm{nr}}\right)=k_{\mathrm{r}} / k_{\mathrm{p}} \approx 0.04$. This value of fluorescence quantum yields places NP-TRCNE complex among moderately fluorescing EDA complexes - much stronger than TCNE complexes for which quantum yield of CT fluorescence at such experimental conditions (nonpolar solvent at room temperature) virtually equals to zero (and even in nonpolar but highly viscous solvent at low temperature, of the order of $100 \mathrm{~K}$, quantum yield of fluorescence of TCNE complexes with aromatic hydrocarbon donors is still by one order of magnitude smaller [12] than the above-estimated for NP-TRCNE system). 


\section{Concluding remarks}

The ground state of NP-TRCNE complex seems to be stabilized predominantly by electrostatic and/or Van der Waals type of interactions, with a minor contribution of charge-transfer resonance stabilization. This is due to the lack of symmetry of TRCNE molecule, which results in a permanent electric dipole moment of this molecule. This property seems to govern very specific head-to-face equilibrium geometry of the ground state EDA complex of NP-TRCNE, which probably may be expected also as an equilibrium structure for TRCNE complexes with other aromatic hydrocarbon donors. It is also probable that such structure of the ground state may be the source of variety of orientational isomers. Since, according to the results of the present calculations this type of structure seems to be rather loosely-bound, this may be a signal that CT absorption of all these complexes may exhibit a strong dependence on the solvent and/or the temperature. This, however, remains a subject to a further experimental verification.

On the other hand, the CT excited state of NP-TRCNE system must be considered as a relatively tightly-bound and compact structure, which means that its emission properties in liquid solution can presumably be satisfactorily described in terms of universal (non-specific) interactions with a dielectric medium, at least as long as the solvent is nonpolar or of medium polarity. This observation is also consistent with the previous studies of the kinetics of monomer-exciplex equilibrium [1], which revealed that NP-TRCNE exciplex in nonpolar $n$-hexane solution follows a simple two-state photokinetic scheme, and although its kinetics of formation and relaxation displays a peculiar case of reversed physical meaning of the decay parameters of exciplex fluorescence, it is straightforward, as compared with sometimes very complicated photokinetic schemes of many other exciplexes in liquid solution, or under jet-cooling conditions in molecular supersonic beams [7, 8].

\section{Acknowledgment}

We gratefully acknowledge financial support of this work by the State Committee for Scientific Research under project 5 P03B 13620.

\section{References}

[1] J. Dresner, K. Kwapisz, I. Deperasińska, J. Prochorow, P. Bruni, A. Giardina, G. Tosi, Acta Phys. Pol. A 102, 443 (2002). Part I of this series.

[2] C.L. Dickinson. D.W. Wiley, B.C. McKusick, J. Am. Chem. Soc. 82, 6132 (1960).

[3] P. Bruni, G. Tosi, unpublished results.

[4] R.S. Mulliken, J. Am. Chem. Soc. 72, 600 (1950); 74, 811 (1952).

[5] E. Gawęda, J. Prochorow, Chem. Phys. Lett. 30, 155 (1975).

[6] J. Prochorow, J. Mol. Struct. 404, 199 (1997).

[7] J. Prochorow, I. Deperasińska, J. Mol. Struct. 450, 47 (1998). 
[8] I. Deperasińska, J. Prochorow, J. Dresner, J. Luminesc. 79, 65 (1998).

[9] R.E. Merrifield, W.D. Phillips, J. Am. Chem. Soc. 80, 2778 (1957).

[10] J. Prochorow, Bull. Acad. Pol. Sci., Ser. Sci. Math. Astr. Phys. XV, 37 (1967).

[11] J. Prochorow, A. Tramer, J. Chem. Phys. 47, 775 (1967).

[12] J. Prochorow, J. Luminesc. 9, 131 (1974).

[13] T.D. Russell, D.H. Levy, J. Phys. Chem. 86, 2718 (1982).

[14] K. Kulinowski, I.R. Gould, N.S. Ferris, A.B. Myers, J. Phys. Chem. 99, 17715 (1995)

[15] Y. Shirota, I. Ysushi, H. Mikawa, Bull. Chem. Soc. Jpn. 47, 991 (1974).

[16] G. Jones II, W.G. Becker, J. Am. Chem. Soc. 105, 1276 (1983).

[17] R. Foster, Organic Charge-Transfer Complexes, Academic Press, London 1969.

[18] N. Mataga, Y. Kanda, T. Asahi, H. Miyasaka, T. Okada, T. Kakitani, Chem. Phys. 127, 239, 249 (1988).

[19] N. Mataga, H. Miyasaka, Prog. Reaction Kinetics, Vol. 19, Elsevier Science Ltd., London 1994, p. 317.

[20] R.S. Mulliken, W.B. Person, Molecular Complexes, A Lecture and Reprint Volume, Wiley Intersince, New York 1969.

[21] J.E. Frey, A.M. Andrews, S.D. Combs, S.P. Edens, J.J. Pukett, R.E. Seagle, L.A. Torreano, J. Org. Chem. 57, 6460 (1992).

[22] K.N. Houk, L.L. Munchausen, J. Am. Chem. Soc. 98, 937 (1976).

[23] N.R. Brinkmann, J.C. Rienstra-Kiracofe, H.F. Schafer III, Mol. Phys. 99, 663 (2001).

[24] G. Briegleb, J. Czekalla, G. Reuss, Z. Phys. Chem. Frankf. Ausg. 30, 316 (1961).

[25] M.J. Mobley, K.E. Rieckhoff, E.-M. Voigt, J. Phys. Chem. 82, 2005 (1978).

[26] R.M. Williams, S.C. Wallwork, Acta Crystallogr. 22, 899 (1967).

[27] M.J.S. Dewar, E.G. Zoebisch, E.F. Healy, J.J.P. Stewart, J. Am. Chem. Soc. 107, 3902 (1985).

[28] P. Hobza, R. Zahradnik, Weak Intermolecular Interactions in Chemistry and Biology, Academia, Prague 1980, p. 157.

[29] M. Kroll, M.L. Ginter, J. Phys. Chem., Ithaca 69, 3671 (1965).

[30] M. Kroll, J. Am. Chem. Soc. 90, 1097 (1968).

[31] M.J. Mantione, Theor. Chim. Acta (Berl.) 15, 141 (1969).

[32] I. Deperasińska, J.S. Kwiatkowski, L. Smentek, Acta Phys. Pol. A 44, 57 (1973).

[33] N. Mataga, T. Kubota, Molecular Interactions and Electronic Spectra, M. Dekker, New York 1970, p. 371.

[34] H. Beens, A. Weller, in: Organic Molecular Photophysics, Ed. J.B. Birks, Wiley, London 1976, p. 159.

[35] J. Prochorow, A. Tramer, J. Chem. Phys. 44, 4545 (1966).

[36] M. Klessinger, J. Michl, Excited States and Photochemistry of Organic Molecules, VCH Publ. Inc., New York 1995, p. 245. 